\title{
Rhinorrhea, CTCAE
}

National Cancer Institute

\section{Source}

National Cancer Institute. Rhinorrhea, CT CAE. NCI Thesaurus. Code C143242.

A disorder characterized by excessive mucous secretions draining from the nose. 